\title{
LA CRESCITA DEI POTERI REGOLATIVI NELLA COMUNITÀ EUROPEA
}

di Giandomenico Majone

\section{Introduzione}

La rilevanza conferita dal Trattato di Maastricht al principio di sussidiarietà se da un lato rivela una diffusa preoccupazione circa la tendenza alla crescita dei poteri di regolazione di Bruxelles, solleva anche diversi interrogativi interessanti sotto il profilo teorico. In primo luogo, è possibile una sovra-regolazione al livello europeo, nonostante che i governi nazionali siano fortemente rappresentati ad ogni livello del processo decisionale? In secondo luogo, se è vero che gli stati membri si sforzano di preservare il più ampio margine possibile di sovranità e di autonomia nel policy-making, come dimostrano le forti resistenze agli interventi comunitari in aree quali la politica macroeconomica e la tassazione indiretta, perché allora essi hanno accettato molte misure regolative non previste dai trattati originari e non strettamente necessarie per il funzionamento del mercato comune? Infine, per quello che riguarda la qualità più che la quantità delle regole comunitarie, quanto è davvero possibile l'innovazione in un sistema dove i poteri formali di iniziativa della Commissione, così come le sue funzioni esecutive, sembrano essere così severamente controllati?

Circa la determinazione degli stati membri nel porre dei limiti alla discrezionalità della Commissione ad ogni livello del policy-making non ci sono dubbi. L'iniziativa politica viene dai capi di Stato o di Governo (Consiglio Europeo); la mediazione ha luogo nel quadro del comitato dei rappresentanti permanenti degli stati membri (COREPER); l'adozione formale delle politiche è prerogativa del Consiglio dei Ministri; l'implementazione è nelle mani delle amministrazioni nazionali. Prima della adozione finale da parte del Consiglio, una proposta della Commissione viene solitamente discussa all'interno di un gruppo di 
lavoro che comprende essenzialmente burocrati degli stati membri; poi viene sottoposta ad un comitato consultivo che include esperti del settore, trasmessa al COREPER per essere discussa da un gruppo di lavoro di rappresentanti nazionali creato all'uopo, rivista ancora una volta dallo stesso COREPER e finalmente spedita al Consiglio per l'approvazione.

La discrezionalità della Commissione nell'esecuzione delle direttive del Consiglio è stata rigidamente regolata dalla Decisione del Consiglio n. 373 del 13 luglio 1987 sul «sistema dei comitati». Tale sistema consiste in un grande numero di comitati associati alla Commissione nell'esercizio delle sue funzioni esecutive. Con il passare degli anni il sistema è diventato sempre più complesso ed include sia comitati consultivi che di altra natura (i cosiddetti comitati di gestione o regolativi). I comitati regolativi e, in qualche misura, anche quelli di gestione possono sospendere una misura presa dalla Commissione e trasmettere il caso al Consiglio che può annullare la decisione della Commissione.

Non è sorprendente che molti studiosi dell'integrazione europea abbiano concluso che le innovazioni di policy nella Comunità Europea sono possibili soltanto quando le preferenze nazionali convergono verso un nuovo approccio. Gli autori di orientamento intergovernativo, in particolare, ritengono si applichi un modello di contrattazione basata sul minimo comun denominatore, una sorta di teoria ricardiana del policy-making comunitario. Così come nella teoria della rendita economica di Ricardo, per la quale il prezzo di un bene è determinato dal costo dell'unità prodotta dall'impresa marginale, secondo questi autori la qualità delle decisioni nella Comunità Europea è determinata dalle preferenze del governo meno favorevole (o marginale). Perciò, fatte salve circostanze eccezionali, l'outcome del processo convergerà verso una posizione di minimo comun denominatore.

Anche altri studiosi che non appartengono alla scuola di pensiero intergovernativo hanno negato la possibilità di una autentica innovazione di policy. Secondo loro la Comunità potrebbe al massimo sperare di «generalizzare e diffondere soluzioni adottate in uno o più stati membri estendendole all'intera comunità. Le soluzioni dei primi stati generalmente determinano il quadro delle soluzioni comunitarie» (Rehbinder e Stewart 1985, 213).

Neppure i neo-funzionalisti hanno ritenuto necessario intro- 
durre una teoria della innovazione di policy. Ernst Haas spiegava la crescita delle competenze comunitarie in termini di «logica espansiva dell'integrazione settoriale» (Haas 1958). Questo autore ipotizzava l'esistenza di un processo di spillover funzionale in forza del quale la decisione iniziale dei governi di delegare i poteri di policy-making in taluni settori ad una istituzione sovranazionale avrebbe creato, inevitabilmente, pressioni per espandere l'autorità di quella stessa istituzione nelle aree di policy contigue. L'economia e la tecnologia, più che la domanda politica o l'iniziativa decisionale, avrebbero guidato il risultato. Versioni più recenti del neo-funzionalismo mostrano una maggiore consapevolezza della crescente importanza dell'innovazione nel sistema decisionale della Comunità. Così, la nozione di spillover politico (George 1993) enfatizza il ruolo delle istituzioni sovranazionali e degli attori sub-nazionali nel processo di spillover funzionale. Tali osservazioni empiriche non sono state tuttavia sviluppate in una spiegazione basata sulle teorie generali del comportamento istituzionale.

Nel tentativo di fornire delle spiegazioni teoriche e non soltanto ad hoc della crescita apparentemente inarrestabile dell'attività regolativa comunitaria, mi è parso utile distinguere una serie di differenti dimensioni della crescita delle politiche: crescita quantitativa; sviluppo della complessità tecnica; espansione dei compiti; innovazione. L'utilità di questa distinzione analitica è dovuta al fatto che fattori causali piuttosto diversi operano nelle varie dimensioni.

Nel secondo paragrafo di questo articolo sono presentati alcuni esempi ed evidenze empiriche relativi alla crescita quantitativa e qualitativa dell'attività regolativa della Comunità Europea negli anni recenti. L'importanza cruciale dell'attività di regolazione nel policy-making comunitario è spiegata nel terzo paragrafo per mezzo di un modello che vede tra le sue variabili principali l'entità limitata del bilancio della Comunità e la scarsa credibilità di accordi puramente intergovernativi sul piano regolativo. Nel quarto paragrafo discuto le dinamiche della delega e del controllo, mentre nel quinto viene presentata una rassegna di alcune recenti teorie sulla entrepreneurship in materia di politiche pubbliche. Il sesto paragrafo evidenzia alcune implicazioni positive e normative ricavabili sulla base dell'analisi precedente. Infine, l'articolo si chiude con alcuni commenti sul tema della riforma istituzionale. 


\section{Alcuni esempi}

Ciascuno degli interrogativi sollevati nell'introduzione si appoggia su un insieme di prove empiriche troppo esteso per essere richiamato in questa sede; soltanto alcuni esempi tra i più significativi saranno presentati qui. Riguardo al fenomeno della over-regulation, si può menzionare la crescita quasi esponenziale del numero di direttive e regolamenti prodotti, in media annuale, dagli organi comunitari: 25 direttive e 600 regolamenti fino al 1970, 50 direttive e 1.000 regolamenti fino al 1975, 80 direttive e 1.500 regolamenti fino al 1985 .

Per fornire un semplice metro di comparazione, nel 1991 Bruxelles produsse 1.564 direttive e regolamenti contro le 1.417 «unità» di legislazione (leggi, ordinanze, decreti) emesse da $\mathrm{Pa}$ rigi. In altre parole, ai giorni nostri, la Comunità introduce nel corpus normativo della Francia più regole di quelle create dalle autorità nazionali. Inoltre, secondo alcune stime, soltanto dal 20 al 25\% dei testi normativi applicabili in Francia sono oggi prodotti dal Parlamento o dal Governo in completa autonomia, cioè senza nessuna precedente consultazione con le istituzioni di Bruxelles (Conseil d'Etat 1992). Sembra dunque che la citatissima predizione di Jacques Delors, secondo la quale entro il 1988 l'80\% della legislazione sociale ed economica avrebbe avuto una origine comunitaria, era forse imprudente sul piano politico, ma non mancava assolutamente di supporto empirico.

Riassumendo alcuni dati statistici, il Consiglio di Stato francese parla di deriva normativa (dérive normative) e di legislazione rigogliosa (droit naturellement foisonnant), mettendo in dubbio la capacità dei governi nazionali di prevedere un simile sviluppo. Nel documento si sostiene, peraltro, che gli stessi stati membri che deplorano la furie réglementaire delle autorità comunitarie sono tra le maggiori cause della over-regulation, un punto questo che analizzeremo più a fondo nelle prossime pagine.

Per quanto riguarda la continua espansione dell'agenda delle istituzioni comunitarie, un indicatore interessante è rappresentato dal numero di Consigli dei Ministri specializzati, che passa da 14 nel 1984 a 21 nel 1993. In aggiunta ai tradizionali Consigli dei Ministri dell'economia, delle finanze, dell'agricoltura, del commercio e dell'industria, abbiamo, negli ultimi anni, incontri regolari tra i Ministri dell'ambiente (dal 1974), dell'istruzione (dal 1974), della ricerca (1975), dei problemi dei 
consumatori (dal 1983), della cultura (dal 1984), del turismo (dal 1988), della protezione civile (dal 1988) e delle telecomunicazioni (dal 1988).

Di sette importanti aree di politiche comunitarie in forte espansione - politiche regionali, ricerca e sviluppo tecnologico, ambiente, protezione del consumatore, istruzione, politica culrurale e audio-televisiva, politica della salute e della sicurezza nel lavoro - soltanto l'ultima era menzionata nel trattato di Roma e soltanto come area decisionale dove la Commissione avrebbe dovuto promuovere una stretta cooperazione tra gli stati membri (art. 118). Nel caso della protezione ambientale, per esempio, tre programmi di azione furono approvati prima che l'Atto Unico Europeo riconoscesse la competenza delle Comunità in tale area. Nel primo programma d'azione (19731976) mancavano proposte definite e ci si concentrava invece su principi generali, mentre i documenti successivi sono diventati sempre più specifici. Il secondo programma (1977-1981) indicava quattro principali aree di intervento, mentre il terzo (1982-1986) sottolineava l'importanza della valutazione dell'impatto ambientale e degli strumenti economici per implementare il principio del «chi inquina paga». A tali documenti hanno fatto seguito concrete azioni: il numero di direttive/decisioni in materia ambientale è infatti salito dalle 10 del 1975, alle 13 del 1980, alle 20 del 1984, alle 25 del 1985 ed alle 17 dei sei mesi che precedettero l'adozione dell'Atto Unico Europeo.

Consideriamo ora l'autentica innovazione in modo distinto rispetto alla mera crescita quantitativa o all'espansione delle mansioni. Come ricordato precedentemente, molti, forse la maggior parte degli studiosi del policy-making europeo, sostengono che le politiche pubbliche della comunità non possono discostarsi da soluzioni di minimo comun denominatore, a meno che gli interessi dei più importanti stati membri non favoriscano qualche nuovo approccio. Così, secondo le interpretazioni degli studiosi di orientamento intergovernativo, in merito al programma per l'attuazione del mercato interno, l'enfasi del Libro Bianco del 1985 (COM 1985/310) sul mutuo riconoscimento rifletterebbe un cambiamento delle preferenze degli stati membri nella direzione di politiche economiche meno interventiste e conseguentemente uno spostamento verso programmi di deregulation (Keohane e Hoffman 1990, 288). Partendo dallo stesso assunto secondo cui le decisioni delle istituzioni sovranazionali rispecchiano le preferenze politiche degli attori politici 
ed economici più potenti in Europa, altri autori hanno sostenuto che la sentenza sul Cassis di Digione, che dava prominenza al principio di mutuo riconoscimento, fosse fondata sull'accoglimento, da parte della Corte di Giustizia Europea, dell'interesse degli stati membri più influenti (Garret 1992; Garret e Weingast 1993).

Una più attenta analisi della realtà empirica rivela un quadro alquanto diverso. In primo luogo, non c'è alcuna prova del fatto che, nel giustificare la decisione riguardo al Cassis, la Corte di Giustizia stesse seguendo qualcosa di diverso dalle proprie convinzioni. Negli argomenti dei difensori dei ricorrenti o nelle osservazioni della Commissione e nelle stesse conclusioni dell'Avvocato Generale non c'è alcuna menzione dell'idea di mutuo riconoscimento (Dehousse 1994). Inoltre, paesi con elevati standard sanitari e di sicurezza, come Francia e Germania, si rendevano conto che un mutuo riconoscimento di tali standard avrebbe implicato una competizione tra i regolatori nazionali. La competizione regolativa, si temeva, avrebbe creato le condizioni per un «dumping sociale» poiché ogni nazione avrebbe tentato di ottenere vantaggi per le proprie industrie o di attrarre investimenti esteri abbassando le soglie dei vincoli regolativi. Pertanto i paesi con sistemi di regolazione sociale avanzati tendevano piuttosto a sostenere il metodo tradizionale della armonizzazione degli standard nazionali più che il principio del mutuo riconoscimento (Alter e Meunier-Aitsahalia 1993).

In effetti era la Commissione, più che gli stati membri, ad avere forti motivi per favorire la riforma dell'approccio tradizionale dell'armonizzazione. Dall'inizio degli anni ottanta, se non prima, era divenuto chiaro che il tentativo di ottenere un mercato integrato armonizzando migliaia di leggi e regolamenti di sei, poi nove ed infine dodici paesi a livelli diversi di sviluppo economico e con tradizioni piuttosto differenti sotto il profilo legale, amministrativo e culturale, era destinato al fallimento. Un nuovo approccio era evidentemente necessario. Già nell'autunno del 1979, il Commissario per il mercato interno suggerì di fronte al Parlamento Europeo che la politica di armonizzazione avrebbe dovuto prendere una direzione diversa, basata sulla sentenza relativa al Cassis. Nel luglio 1980, la Commissione mandò agli stati membri, al Parlamento Europeo ed al Consiglio, una «comunicazione interpretativa» con la quale si dichiarava inequivocabilmente che la sentenza sul Cassis avrebbe guidato il nuovo approccio al problema dell'armonizzazione. 
Gli stati membri reagirono preoccupati per le ampie conseguenze delineate dalle dichiarazioni della Commissione, in particolare per la prospettiva di una diretta competizione tra $\mathrm{i}$ regolatori nazionali. L'ufficio legale del Consiglio redasse una contro-interpretazione del caso, sostenendo che la generalizzazione operata dalla Commissione rispetto all'argomentazione della Corte era eccessiva e concludendo che la sentenza sul Cassis di Digione non aveva cambiato praticamente nulla (Alter e Meunier-Aitsahalia 1993). Alla fine, tuttavia, prevalse l'interpretazione della Commissione. Al meeting di Milano, nel giugno 1985, gli stati membri approvarono il Libro Bianco e la sua strategia di mutuo riconoscimento.

Anche per quel che riguarda il policy-making «quotidiano», il punto di vista prevalente sembra essere quello secondo cui la Commissione potrebbe, al massimo, diffondere nella Comunità le soluzioni adottate dai più avanzati degli stati membri. Si ammette tuttavia che ci siano anche esempi di autentica innovazione di policy. Così la direttiva sul biphenyl-policlorinato (PCB) (1976/769) «non trovava alcun parallelo nei regolamenti di uno stato membro», mentre la direttiva sui valori limite per il diossido di zolfo (1980/779) stabiliva, su base comunitaria, standard di qualità ambientali che la maggior parte degli stati membri non avevano sino ad allora adottato come strategia di controllo (Rehbinder e Stewart 1985, 214). Comunque, in mancanza di spiegazioni teoriche adeguate, esempi simili tendono ad essere interpretati come casi speciali senza alcuna significatività generale.

Un orientamento così semplicistico non sembra essere più sostenibile. L'Atto Unico Europeo, attraverso l'introduzione di maggioranze qualificate, non soltanto per la legislazione sul mercato interno ma anche per altre importanti aree della regolazione sociale, ha creato condizioni favorevoli per lo sviluppo di straordinarie innovazioni regolative. Per esempio la direttiva cornice 1989/391 su salute e sicurezza nel lavoro va persino al di là della filosofia e della pratica di una nazione avanzata come la Germania (Feldhoff 1992). Tra le caratteristiche più importanti di questa direttiva spiccano la sua estensione (è applicabile a tutti i settori di attività, pubblica e privata, inclusi i servizi, la scuola, la cultura e il tempo libero), i vincoli generali imposti agli imprenditori, l'esigenza di informazione per il lavoratore e l'enfasi posta sulla sua partecipazione.

Ugualmente innovative sono la direttiva sugli impianti indu- 
striali $(1989 / 392)$ e, in una sfera più limitata, la direttiva 1990/ 270 su salute e sicurezza nel lavoro davanti a schermi televisivi. Entrambe le direttive si riferiscono ad un concetto di «ambiente di lavoro» che apre la possibilità di interventi regolativi in un settore tradizionalmente considerato fuori dal campo della sicurezza professionale, e considerano fattori psicologici, stress e fatica come elementi importanti di cui tener conto in un approccio regolativo moderno. È difficile trovare principi egualmente avanzati nella legislazione di qualsiasi potenza industriale, dentro e fuori l'Unione Europea.

Per spiegare simili output decisionali ci servono teorie nuove e più analitiche sul processo di policy nella Comunità. Tali teorie devono essere in grado di spiegare la trasformazione qualitativa della regolazione comunitaria come pure la sua crescita apparentemente inarrestabile.

\section{Un modello del policy-making regolativo}

Per capire il policy-making comunitario si deve partire dalle dimensioni del bilancio comunitario, piuttosto ridotte nonostante la significativa crescita degli ultimi anni. Esso rappresenta infatti meno del $4 \%$ di tutta la spesa dei governi centrali degli stati membri e meno dell' $1,3 \%$ del prodotto nazionale lordo dell'Unione. In confronto, dai governi nazionali viene speso dal 45 al $50 \%$ della ricchezza prodotta dagli stati membri. Il budget comunitario non è soltanto esiguo ma anche rigido: almeno il $70 \%$ degli stanziamenti consiste in fondi di spesa obbligatoria per programmi quali la sezione di garanzia del Fondo Europeo di Orientamento e Garanzia Agricola (FEOGA).

In secondo luogo, è importante distinguere tra politiche regolative e politiche che implicano direttamente la spesa di stanziamenti pubblici. Esempi di quest'ultimo tipo sono le politiche redistributive, che trasferiscono risorse da un gruppo di individui, regioni o nazioni ad un altro gruppo, oppure politiche distributive come i lavori pubblici o il sostegno finanziario per la ricerca e lo sviluppo tecnologico, che allocano risorse pubbliche tra differenti attività. Ora, una importante caratteristica del policy-making regolativo è rappresentata dallo scarso impatto delle limitazioni di bilancio sull'attività dei regolatori. L'entità dei programmi diretti di spesa è vincolata agli stanziamenti di bilancio e, in definitiva, alla dimensione delle entrate 
fiscali dei governi. Al contrario i costi reali di molti programmi regolativi sono sopportati dalle aziende e dagli individui che devono conformarsi ad essi. Comparate con tali costi, le risorse che servono a produrre le regolazioni sono insignificanti. Il significato di questa differenza tra politiche regolative e politiche di spesa diretta non è irrilevante. La distinzione è importante specialmente per l'analisi del policy-making comunitario perché non soltanto i costi economici, ma anche quelli politici e amministrativi dell'implementazione dei regolamenti e delle direttive comunitarie, sono sopportati, direttamente o indirettamente, dagli stati membri.

In terzo luogo si deve assumere che la Commissione Europea, come ogni altra organizzazione burocratica, tenti di massimizzare la sua influenza nei limiti consentiti dai vincoli di bilancio, politici e legali. In questa sede mi concentrerò sui vincoli di bilancio. Come già notato, le risorse finanziarie della Comunità sono riservate, per la maggior parte, alla politica agricola e ad una serie di programmi distributivi e redistributivi. Le risorse rimanenti sono insufficienti per sostenere iniziative su larga scala in aree come la politica industriale, l'energia, la ricerca o l'innovazione tecnologica. Perciò, l'unico modo per la Commissione di accrescere il proprio ruolo è quello di espandere le proprie attività regolative, anche oltre l'ambito richiesto per il funzionamento del mercato comune.

Come si è visto nella sezione precedente, questa strategia si è rivelata molto felice, anche troppo secondo alcuni, ma le ragioni di tale successo non possono essere trovate soltanto nelle preferenze della Commissione. Il sistema di policy-making comunitario include molti attori: industriali, sindacalisti, gruppi di interesse, politici, burocrati nazionali e sovranazionali, esperti indipendenti e così via. Abbiamo visto come la Commissione giochi un ruolo cruciale sul lato dell'offerta della regolazione comunitaria. Adesso invece dobbiamo osservare il lato della domanda. Per semplificare l'esposizione, considererò soltanto gli attori più importanti su questo lato, ovvero i governi nazionali (per una analisi più dettagliata cfr. Majone 1992 e 1994a).

Può sembrare illogico, se non completamente sbagliato, discutere il ruolo degli stati membri nello sviluppo della regolazione comunitaria dal lato della domanda. Dopo tutto, molti degli atti con valore normativo devono essere approvati dal Consiglio, che rappresenta gli interessi dei governi nazionali ed è considerato il legislatore reale nel sistema europeo. Perché al- 
lora non porre gli stati membri e la Commissione sullo stesso lato della equazione di domanda e offerta come «co-produttori» degli output regolativi? Sebbene questa interpretazione sia formalmente corretta, molti fattori suggeriscono che dal punto di vista del policy-making è più conveniente considerare, con riferimento alla regolazione comunitaria, i governi nazionali sul lato della domanda più che su quello dell'offerta.

In primo luogo è piuttosto evidente che molte delle proposte della Commissione sono avanzate sulla base dell'iniziativa degli stati membri (o di altri attori come il Parlamento Europeo, il Consiglio dei Ministri, la Commissione Economica e Sociale, i rappresentanti di interessi privati). Per esempio i governi tedesco ed olandese hanno giocato un ruolo importante nella formulazione e poi nella messa a punto delle direttive comunitarie riguardanti il controllo sullo scarico dei veicoli, mentre il governo britannico ha esercitato pressioni evidenti sulla Commissione per liberalizzare il mercato delle assicurazioni sulla vita e non, un settore dove le compagnie britanniche godono di un netto vantaggio sui loro competitori del continente. Secondo il rapporto del Consiglio di Stato francese citato sopra, delle ultime 500 proposte di regolazione e direttive presentate dalla Commissione nel 1991, soltanto il 6\% figura come «iniziative spontanee». Dunque, una schiacciante maggioranza delle proposte sarebbe davvero prodotta dalla domanda degli stati membri o di altri attori.

Un secondo fattore, teoricamente più importante, è relativo alla credibilità della policy. Come già detto nell'introduzione, non è così ovvio a priori che gli stati membri vogliano delegare i poteri regolativi estendendoli oltre quanto previsto dai trattati o dalla logica dell'allargamento funzionale in un mercato sempre più integrato. Come ha mostrato in un famoso articolo Ronald Coase (1960), la presenza di esternalità negative non garantisce di per sé un effettivo coordinamento tra attori indipendenti.

Una significativa implicazione del teorema di Coase è il fatto che la ragione fondamentale per una regolazione sovranazionale è il fallimento regolativo più che quello del mercato. I fallimenti del mercato che abbiano conseguenze internazionali, per esempio l'inquinamento oltre frontiera, potrebbero essere affrontati ricorrendo a modalità cooperative (cioè intergovernative), e quindi senza la necessità di delegare poteri regolativi ad un organo sovranazionale, a patto che: i regolatori nazionali ab- 
biano la volontà e la capacità di considerare le ripercussioni internazionali delle loro scelte; abbiano sufficienti conoscenze delle intenzioni altrui; i costi di transazione dell'organizzazione e del monitoraggio della cooperazione non siano troppo alti e, soprattutto, ognuno possa fidarsi degli altri attori e della loro buona fede nell'implementazione delle decisioni congiunte.

I fallimenti regolativi internazionali si manifestano invece quando una o più di queste condizioni non sono soddisfatte. Per esempio, è difficile di solito stabilire se gli accordi regolativi intergovernativi sono veramente osservati o no. Questo perché molte delle regole economiche e sociali sono discrezionali. Poiché i regolatori mancano dell'informazione che soltanto le aziende regolate hanno, e poiché i governi sono riluttanti, per motivi politici, ad imporre costi eccessivi all'industria, la contrattazione è una caratteristica essenziale del processo di applicazione delle politiche regolative. Al di là di ciò che la legge dice, il processo di regolazione non è semplicemente un processo nel quale i regolatori decidono ed i regolati obbediscono. Si crea invece un «mercato» riguardo a quali siano esattamente $i$ doveri da parte di questi ultimi (Peacock 1984). Poiché la contrattazione è così pervasiva, può essere impossibile per un osservatore esterno determinare se una regolazione internazionale sia stata, di fatto, violata.

Quando è difficile osservare se i governi stiano facendo un onesto sforzo teso al mantenimento dell'accordo cooperativo, l'accordo stesso non è più credibile. Una soluzione è appunto quella di delegare alcune mansioni regolative ad autorità sovranazionali con poteri di monitoraggio o di imposizione di sanzioni. Qualche volta i problemi di credibilità di un governo non riguardano tanto i rapporti con gli altri esecutivi coinvolti nell'accordo quanto i rapporti con terze parti come le aziende regolate. Così, quando l'inquinamento ha effetti internazionali e le sanzioni determinano svantaggi competitivi rilevanti per le aziende presenti sul mercato internazionale, queste saranno portate a credere che i regolatori nazionali non vorranno applicare tali sanzioni in maniera altrettanto rigorosa quando decidono in modo unilaterale e quando agiscono sotto la supervisione sovranazionale. Per questo il trasferimento di poteri regolativi ad una autorità sovranazionale come la Commissione Europea, rendendo più credibile una regolazione severa, può migliorare il comportamento delle aziende regolate (Gatsios e Seabright 1989). Inoltre la Commissione, essendo coinvolta nella 
regolazione di un gran numero di aziende in tutta l'Unione Europea, ha molto più da guadagnare rispetto ai regolatori nazionali da una azione risoluta in ogni caso individuale: una applicazione debole delle norme potrebbe distruggere la sua credibilità agli occhi di un numero maggiore di aziende. Così essa può essere più disposta degli stati membri ad imporre sanzioni, anche se i suoi costi e benefici diretti nel fare ciò non sono molto differenti (Gatsios e Seabright 1989, 50). Il fatto che la Commissione sia coinvolta nella regolazione di un gran numero di aziende attraverso l'Europa spiega anche perché essa sia meno vulnerabile rispetto al rischio della «cattura regolativa» da parte dei regolatori nazionali.

Probabilmente il più grande vantaggio della membership europea in un periodo di cambiamenti di grande portata è la possibilità di delegare decisioni politicamente difficili (come per esempio l'eliminazione dell'aiuto pubblico all'industria, l'imposizione di regole per la competizione, la liberalizzazione degli scambi e l'applicazione delle normative ambientali in una fase di recessione economica) ad istituzioni sovranazionali non maggioritarie (Majone 1994b). Mostrando che le loro scelte sono vincolate alla appartenenza europea, gli stati membri possono accrescere la credibilità internazionale delle loro politiche ed al tempo stesso ridurre il potere delle coalizioni redistributive interne. In sostanza, la scarsa credibilità di accordi puramente intergovernativi, insieme al vantaggio recato ai governi dal trasferimento di decisioni difficili ad una istituzione non maggioritaria, spiega la disponibilità degli stati membri di delegare importanti poteri regolativi alla Commissione. Nel prossimo paragrafo esploreremo le conseguenze di questo processo.

\section{La dinamica della delega e del controllo}

La delega di estesi poteri giudiziari e di policy-making alle istituzioni sovranazionali è ciò che distingue la Comunità Europea rispetto ai regimi internazionali tradizionali. Le implicazioni di questo fenomeno non sono tuttavia ancora pienamente comprese. Né i neo-funzionalisti né tantomeno gli studiosi di orientamento intergovernativo hanno seriamente considerato la dinamica della delega e del controllo; i primi a causa della loro fede negli automatismi dello spillover funzionale, i secondi in virtù dell'assunto secondo cui le istituzioni sovranazionali produrreb- 
bero semplicemente una fedele e pacifica trasposizione degli interessi nazionali nelle politiche. Per analizzare le conseguenze della delega di poteri di policy-making e capire le possibilità di un controllo politico dobbiamo utilizzare, più che i tradizionali lavori sull'integrazione europea, la letteratura sulla relazione politica-burocrazia e quella sulle relazioni principale-agente.

Le più recenti ricerche sulle relazioni politica-burocrazia convergono sulla constatazione che quest'ultima ha un livello di autonomia significativo, mentre il controllo politico diretto è piuttosto debole (Wilson 1980; Moe 1987; Majone 1994c). Un serio controllo sulle politiche risulta piuttosto costoso in termini di tempo e risorse, e difficile da compiere con accuratezza in condizioni di incertezza e complessità. In ogni caso, i parlamentari sono più preoccupati di soddisfare gli elettori, aumentando così le proprie possibilità di rielezione, che non di controllare la burocrazia. Il risultato è che normalmente essi non investono le loro scarse risorse in un controllo generale delle politiche. Invece, preferiscono intervenire in maniera più rapida e puntuale, ed in modo non dispendioso, per proteggere clienti particolari in materie particolari (Mayhew 1974). Perciò il controllo parlamentare è poco coordinato e frammentato. Analogamente, la letteratura sul processo di bilancio ha formulato seri dubbi sull'immagine del budget come un effettivo strumento di controllo. Già nel 1964 Wildavsky scopriva che il budgeting è un processo decentralizzato e incrementale che si traduce in crescite automatiche il cui effetto è isolare ulteriormente la burocrazia dal controllo politico.

Le nuove teorie basate sul modello principale-agente danno una valutazione più positiva delle possibilità di un controllo politico della burocrazia. Secondo tali teorie il controllo è possibile poiché sono i politici eletti a creare le burocrazie. Essi disegnano istituzioni amministrative con strutture di incentivi che facilitano il controllo e monitorizzano l'attività burocratica in modo da bilanciare le asimmetrie informative. Così, alcune teorie basate su questo modello, come le recenti versioni delle teorie intergovernative (Moravcsik 1993), suppongono l'esistenza di decisori centrali ben informati che modellano in modo sistematico le preferenze degli agenti burocratici e sono in grado di esercitare un controllo politico razionale (Wood e Waterman 1991, 803).

Tuttavia il processo è considerevolmente più complesso di quello delineato da tali teorie. Nella fase della delega, i princi- 
pali politici sono certamente liberi di selezionare i loro agenti e di imporre a questi ultimi una struttura di incentivi rilevante per il loro comportamento. Nel tempo, tuttavia, i burocrati accumulano una competenza specifica e questa asset specificity (Williamson 1985) altera la relazione originaria. Ora i politici devono trattare con gli agenti da loro stessi selezionati, ma in questo rapporto i burocrati hanno acquisito un vantaggio in termini tecnici ed operativi. Il risultato è dunque che questi ultimi sono sempre più in grado di perseguire i propri obbiettivi in grande autonomia. Come scrive Terry Moe (1987, 143):

Una volta che un'agenzia è stata creata, la realtà politica si trasforma. I burocrati sono adesso attori politici in piena regola: hanno interessi di carriera ed istituzionali che possono non essere pienamente congruenti con le loro mansioni formali; hanno risorse di potere - expertise e competenze delegate che possono essere impiegate per fini «egoistici». Sono diventati giocatori i cui interessi e le cui risorse alterano il gioco politico.

La ricerca più recente sulle relazioni tra burocrati e politici getta una luce importante sulla dinamica della delega e del controllo nel contesto della Comunità Europea. Anche per i rappresentanti degli stati membri che si siedono nel Consiglio dei Ministri un serio controllo sulle politiche è dispendioso, in termini di risorse e di tempo, nonché difficile da realizzare compiutamente. $\mathrm{Da}$ ciò la loro scarsa disponibilità ad investire molte risorse in tali attività. Come si è detto nel secondo paragrafo, il sistema dei comitati è un tentativo di controllare la discrezionalità della Commissione nell'esecuzione delle direttive del Consiglio. I comitati regolativi e di gestione creati nell'ambito di questo sistema possono bloccare una misura della Commissione e trasmettere il caso al Consiglio, che può dunque rovesciare la decisione della Commissione stessa. Tuttavia anche in questi comitati la Commissione mantiene il ruolo di presidenza e gode per di più di una forte presunzione a proprio favore (Ludlow 1991, 107). Secondo il più dettagliato studio empirico sull'attuale sistema dei comitati, «i funzionari della Commissione non pensano in genere che il loro comitato abbia ridotto significativamente la libertà della Commissione stessa ed ancor meno che sia stato creato per consentire il controllo degli stati membri» (Institut fuer Europaeische Politik 1989, 9). Secondo lo stesso studio, il Consiglio agisce soltanto raramente nelle materie tecniche complesse trattate dai comitati previsti nell'ambito di questo sistema, ma quando lo fa, le sue decisioni appog- 
giano per lo più le proposte originarie della Commissione ( $i b i$ dem, 123). In effetti, la Commissione ha ottenuto un altissimo risultato (98 per cento) di accettazione delle proprie proposte da parte dei vari comitati regolativi (Eichener 1992).

Anche nel caso della iniziativa di politiche, la procedura formale, secondo la quale le proposte della Commissione sono discusse in un gruppo di lavoro che comprende esperti nazionali e poi sottoposte ad un comitato consultivo e riviste infine dal COREPER, dà l'impressione di uno stretto controllo che non corrisponde alla realtà. Quanto si conosce circa il modus operandi dei comitati consultivi e dei gruppi di lavoro suggerisce che le discussioni si basano più su problemi sostantivi che su allineamenti nazionali. Un notevole grado di copinage technocratique si sviluppa tra i funzionari della Commissione e gli esperti nazionali interessati a trovare soluzioni pragmatiche più che a difendere posizioni politiche (Eichener 1992). Quando una proposta della Commissione raggiunge il Consiglio dei Ministri tutti i dettagli tecnici sono stati sviluppati e le ulteriori modificazioni solitamente non riguardano gli aspetti essenziali.

Questo è dovuto in parte al fatto che, sebbene il Consiglio possa supervisionare con i suoi gruppi di lavoro l'attività della Commissione, esso non può competere con l'esperienza a disposizione della stessa Commissione e delle sue Direzioni Generali (Peters 1992, 119). Gli uffici della Commissione responsabili per una particolare area di policy formano il nodo centrale di un vasto issue network che include, oltre agli esperti provenienti dalle amministrazioni nazionali, esperti indipendenti, accademici, rappresentanti di movimenti ambientali, dei consumatori e di altri interessi pubblici, rappresentanti di interessi economici, di organizzazioni professionali e di governi sub-nazionali.

I funzionari della Commissione intraprendono lunghe discussioni con tutti questi attori ma restano alla fine liberi di scegliere quali idee e proposte adottare. La varietà delle posizioni, che è solitamente molto più ampia che a livello nazionale, accresce la libertà di scelta da parte dei burocrati comunitari. Può anche accadere che gli esperti nazionali trovino nella Commissione un'arena più ricettiva per le nuove idee rispetto alle stesse amministrazioni statali. L'importante direttiva sull'Organizzazione (1989/392), già menzionata nel secondo paragrafo, offre un chiaro esempio di ciò. L'allegato tecnico estremamente importante della direttiva fu sviluppato da un esperto sindacale 
britannico che in precedenza aveva cercato di riformare l'approccio inglese in materia di sicurezza nel posto di lavoro. Dopo aver fallito nel tentativo di persuadere i policy-makers nel proprio paese, egli aveva portato le sue idee innovative a Bruxelles, dove furono accolte dai funzionari della Commissione ed alla fine divennero leggi europee (Eichener 1992).

\section{L'imprenditorialità nelle politiche}

L'esistenza di larghi margini di discrezionalità regolativa è una condizione necessaria ma non sufficiente per una autentica innovazione di policy. Dobbiamo considerare anche la capacità dei funzionari della Commissione di giocare il ruolo di «imprenditori» di una data policy. Kingdom (1984) descrive gli imprenditori di policy come costantemente alla ricerca di occasioni da sfruttare per far passare le proprie idee. Finestre di policy si aprono in quelle occasioni, relativamente rare, nelle quali tre flussi solitamente separati - quello dei problemi da risolvere, quello della politica e quello delle risposte di policy - convergono.

Gli imprenditori di policy interessati ad un problema particolare cercano nel flusso delle proposte di policy le soluzioni da abbinare al loro problema e poi cercano di cogliere la recettività politica esistente in dati momenti per spingere le soluzioni del problema.

Un imprenditore di policy di successo possiede tre qualità fondamentali: in primo luogo egli deve essere preso sul serio come esperto, come leader di un gruppo di interesse forte, o come decision maker autorevole; in secondo luogo deve essere noto per le proprie connessioni politiche o per le capacità di negoziazione; infine, ed è probabilmente la cosa più importante, gli imprenditori di successo sono ostinati nella loro azione (Kingdom 1984, 189-90). A causa delle modalità di reclutamento, della strutturazione della carriera e del ruolo cruciale giocato dalla Commissione nell'iniziativa delle politiche, i funzionari della Commissione stessa hanno spesso le qualità di un imprenditore di policy di successo, ad un livello difficilmente raggiungibile dai burocrati nazionali.

In particolare, la Commissione mostra un alto grado di ostinazione nel perseguimento degli obbiettivi. Molte importanti innovazioni di policy nella Comunità sono state ottenute dopo 
lunghi anni durante i quali la Commissione aveva persistito nel suo sforzo di «ammorbidire» l'opposizione degli stati membri, aspettando nel contempo che si aprisse una finestra di opportunità. Un esempio da manuale è il caso del regolamento sul Controllo delle fusioni commerciali, approvato dal Consiglio il 21 dicembre 1989, dopo un braccio di ferro durato oltre venti anni.

Già nel 1965 la Commissione notava come il Trattato di Roma fosse piuttosto carente per la mancanza di poteri di controllo sulle fusioni. L'anno seguente un gruppo di esperti fu incaricato di studiare il problema delle concentrazioni economiche nel mercato comune. La maggioranza del gruppo riteneva che l'art. 85 del Trattato di Roma potesse essere applicato alle fusioni che realizzassero dei monopoli, ma la Commissione scelse di seguire l'opinione contraria, della minoranza. Si accettava tuttavia l'opinione della maggioranza relativamente all'applicabilità dell'art. 86 a quelle fusioni che coinvolgessero una impresa che deteneva già una posizione «dominante» all'interno del mercato comune. La Corte Europea di Giustizia seguì l'interpretazione della Commissione nel caso Continental Can (1973).

All'inizio del 1974 il Parlamento Europeo e la Commissione Economica e Sociale approvarono con larghe maggioranze una proposta per un regolamento di controllo sulle fusioni, ma gli stati membri non erano ancora preparati a concedere alla Commissione i poteri richiesti. Seguì un lungo periodo di inazione, finché il processo non fu nuovamente messo in moto dalla innovatrice sentenza sul caso Philip Morris del 17 novembre 1987, con la quale la Corte di Giustizia stabilì, contrariamente all'opinione giuridica prevalente, che l'art. 85 si doveva applicare all'acquisizione, da parte di una impresa, di una quota azionaria di una ditta competitrice, laddove l'effetto fosse quello di ridurre o distorcere la competizione. La Commissione appoggiò fortemente la decisione della Corte. Era chiaro che si trattava di un nuovo passo importante, dopo il caso Continental Can, verso il controllo sulle fusioni commerciali di portata comunitaria. Nel frattempo, il programma «Europa 1992» per il completamento del mercato interno aveva stimolato ondate di fusioni. Questo sviluppo aprì la finestra di opportunità che la Commissione aveva atteso così a lungo. Ora il controllo centralizzato sulle fusioni di portata comunitaria poteva essere proposto come essenziale per il processo di completamento del mercato interno. Dunque, la convergenza dei tre elementi suggeriti da 
Kingdom, problemi, situazione politica e soluzioni proposte, produsse il regolamento del 1989 relativo al controllo sulle fusioni commerciali.

Questo episodio della storia dei processi decisionali comunitari fornisce un chiaro esempio dell'ostinazione e delle capacità di imprenditorialità politica della Commissione, ma dimostra anche un punto di portata più generale, ovvero che una adeguata spiegazione dello sviluppo delle politiche nella Comunità Europea deve essere basata sulla dinamica dell'intero sistema e prestare attenzione alle relazioni di reciproca dipendenza tra le istituzioni europee. Così, nel secondo paragrafo, abbiamo menzionato il significato strategico del principio di mutuo riconoscimento per la Commissione piuttosto che per gli stati membri. Soltanto tramite questo nuovo approccio ai problemi dell'armonizzazione gli obbiettivi del programma di mercato interno hanno potuto essere ottenuti in tempo. A sua volta questo nuovo approccio era stato reso possibile grazie all'azione e alle decisioni sia della Commissione che della Corte di Giustizia. La relazione di reciproca dipendenza tra queste due istituzioni è stata molto ben espressa da Alter e Meunier-Aitsahalia: «la decisione sul Cassis ha promosso l'idea del mutuo riconoscimento, e l'imprenditorialità della Commissione ha portato il problema sul tavolo sollevando il dibattito. Sia la decisione della Corte che la risposta della Commissione sono state necessarie per produrre la nuova politica di armonizzazione. La decisione giudiziaria era necessaria per incoraggiare la Commissione ad emettere la propria audace comunicazione... La comunicazione della Commissione, tuttavia, $f u$ anch'essa necessaria per portare $i$ contenuti della sentenza nell'arena politica» $(1993,19)$.

Combinando insieme i concetti dalla teoria della scelta pubblica con alcuni studi storici di casi, William Riker fornisce ulteriori elementi di chiarificazione relativamente alle strategie perseguite dagli imprenditori di policy al fine di cambiare le coalizioni politiche esistenti. Egli sostiene che attraverso la formazione dell'agenda, il comportamento strategico, e specialmente con l'introduzione di nuove dimensioni di policy nel dibattito, l'imprenditore politico può spezzare gli equilibri esistenti al fine di creare nuovi e più convenienti outcomes politici. L'imprenditore di successo «sonda il terreno finché non trova nuove alternative, nuove dimensioni che possano entrare in sintonia con le preferenze altrui» (Riker 1986, 64). 
Un buon esempio di questa strategia è l'introduzione da parte della Commissione del concetto di ambiente di lavoro nel dibattito europeo sulla salute e la sicurezza nel lavoro. Come già menzionato nel secondo paragrafo, questo concetto apre la possibilità di interventi regolativi in aree, come l'ergonomica, tradizionalmente considerate esterne al campo della sicurezza nel lavoro. Contro il parere degli approcci intergovernativi, secondo i quali il policy-making comunitario è sotto il controllo degli stati membri più potenti, si deve rilevare che l'importante direttiva sul meccanismo ed altre direttive ugualmente innovative in questo settore decisionale (cfr. secondo paragrafo) sono state ispirate dalla filosofia regolativa di due piccole nazioni, Olanda e Danimarca, che per prime avevano introdotto il concetto di ambiente di lavoro all'interno della propria legislazione, e che invece tali decisioni furono contrastate dalla Germania al fine di preservare i poteri e la logica tradizionale dei propri organismi regolatori (Feldhoff 1992; Eichener 1992).

\section{Implicazioni positive e normative}

Come già indicato in precedenza, per capire la nascita e la crescita delle politiche regolative nella Comunità Europea è importante distinguere $\mathrm{i}$ differenti aspetti del fenomeno: crescita quantitativa, complessità regolativa, espansione dei compiti e «approfondimento», cioè autentica innovazione di policy. Le teorie discusse nelle pagine precedenti suggeriscono alcune osservazioni relative a queste varie dimensioni di sviluppo e crescita.

Il modello di domanda e offerta della regolazione comunitaria che si è sintetizzato nel terzo paragrafo cerca di spiegare le origini della regolazione più che la dinamica corrente del processo. Tuttavia il modello ha implicazioni significative per i temi affrontati in questo articolo. Ricordiamo infatti che le principali variabili esplicative, oltre al desiderio della Commissione di accrescere la propria influenza, sono i vincoli di bilancio e la bassa credibilità degli accordi regolativi intergovernativi.

Paradossalmente, il tentativo da parte degli stati membri di limitare la portata delle politiche sovranazionali, attraverso l'imposizione di un rigido vincolo di bilancio alla Commissione, ha favorito lo sviluppo di un policy-making largamente immune dalla disciplina di bilancio. Come scrive uno studioso americano: 
Le cifre del bilancio e delle entrate sono buoni indicatori di quello che accade nei campi del welfare, nella difesa e nella politica fiscale, e possono essere usate per comunicare efficacemente con il pubblico passando sopra alle interminabili contese sugli specifici programmi tra i vari gruppi di interesse... In un mondo come quello della regolazione invece, dove il governo impone le regole ma praticamente tutto il resto rimane nella sfera dell'economia privata, non possiamo in genere contare su cifre aggregate per descrivere ciò che è stato «tassato» e ciò che è stato «speso» nel perseguimento di una politica pubblica. Invece, abbiamo liste, infinite liste di progetti che il governo vorrebbe che altri intraprendessero (De Muth 1984, 25).

Così, la continua espansione è un carattere strutturale del policy-making regolativo e non soltanto il risultato degli spillover funzionali e della «logica espansiva dell'integrazione settoriale» come sostenuto dai neo-funzionalisti. Due fattori ulteriori contribuiscono alla crescita apparentemente inarrestabile della regolazione europea. Innanzitutto, come già detto in preceden$z a$, la grande maggioranza dei regolamenti comunitari e delle direttive non sono il frutto di iniziative spontanee della Commissione ma sono sollecitati dalle domande provenienti dai singoli stati membri e dal Consiglio, ma anche dal Parlamento Europeo, dalla Commissione Economica e Sociale, dai governi regionali e da vari gruppi di interesse. La possibilità, prevista dal Trattato di Maastricht, per il Parlamento Europeo di chiedere alla Commissione proposte legislative può soltanto rafforzare questo trend.

Mentre la responsiveness della Commissione verso tali richieste può accrescere la sua legittimazione politica, le domande incontrollate e non coordinate possono produrre una serie di conseguenze negative, tra le quali la più ovvia è l'inflazione legislativa. Tali conseguenze sono aggravate da fattori istituzionali. Poiché la Commissione Europea è un organo collegiale, il coordinamento centrale dei programmi regolativi promossi dalle varie Direzioni Generali risulta piuttosto difficile. La mancanza di un coordinamento comporta serie incoerenze all'interno e tra i programmi regolativi, oltre alla mancanza di procedure razionali per la selezione delle priorità ed una insufficiente attenzione per il rapporto costo-efficacia delle singole regole.

Un metodo per ridurre l'eccesso di regolazione consisterebbe nella creazione di una istituzione con il potere di controllare l'intero processo regolativo e di disciplinare l'attività delle Direzioni Generali comparando i benefici sociali delle proposte con i costi imposti all'economia europea. Una tale istituzione, una sorta di «stanza di compensazione regolativa», dovrebbe essere 
posta al più alto livello della Commissione ed avere il potere di chiedere alle Direzioni Generali la trasmissione di bozze annuali dei programmi regolativi da sottoporre a verifica. Qualora emergessero disaccordi o gravi contraddizioni, verrebbe richiesto al Presidente della Commissione o ad un «gruppo di lavoro sulla Regolazione» di intervenire. Questo processo di verifica faciliterebbe il lavoro della Commissione nella selezione delle domande di misure regolative europee e nel modellare un insieme coerente di proposte da sottoporre a Consiglio e Parlamento. L'utilità di questo procedimento sarebbe ulteriormente accresciuta ove si coordinasse il controllo delle proposte regolative con la normale analisi dei documenti di bilancio, in modo da legare il livello degli stanziamenti alla valutazione circa l'efficacia dei diversi programmi (Majone 1992).

Consideriamo adesso il problema della complessità regolativa. Molti studiosi del policy-making europeo hanno osservato che le direttive comunitarie presentano un livello di dettaglio tecnico molto più elevato rispetto a quello della legislazione nazionale comparabile. L'opinione corrente secondo la quale tale livello di complessità è dovuto essenzialmente al perfezionismo tecnico della Commissione manca di plausibilità: la Commissione è infatti molto ristretta rispetto al numero di compiti che ha; le sue risorse sono piuttosto limitate e la sua composizione si basa, per la maggior parte, sui «generalisti» e non su esperti tecnici. È invece, per lo più, la diffidenza degli stati membri ad essere responsabile di tale complessità. I rappresentanti nazionali infatti, dubbiosi circa l'impegno degli altri stati nell'implementare correttamente le decisioni europee e generalmente piuttosto disinformati circa i differenti stili nazionali di amministrazione, insistono nella meticolosa definizione degli obblighi reciproci sino al punto di includere spesso nei testi normativi anche formule matematiche, chimiche o statistiche.

Gli stati membri diffidano non soltanto gli uni degli altri, ma anche della stessa Commissione. Come già notato nel quarto paragrafo essi hanno creato, al fine di limitare la discrezionalità di quest'organo, un complesso sistema di gruppi di lavoro e comitati consultivi controllati da esperti nazionali. Per le ragioni sopra menzionate, il sistema non si è rivelato molto efficace nel ridurre la libertà di scelta della Commissione, ma tende ad introdurre un forte pregiudizio tecnico nel processo regolativo. Questo avviene perché molti esperti nazionali sono estremamente specializzati ed interessati più ai dettagli che al rapporto 
costi-efficacia ed alla facilità di applicazione delle soluzioni. Questo pregiudizio tecnico, combinato con la riluttanza del Consiglio di intraprendere un serio controllo sulla policy e con la mancanza di un monitoraggio generale al livello della Commissione, è probabilmente un ulteriore fattore che contribuisce alla complessità regolativa.

Questa ipotesi è corroborata da considerazioni teoriche più generali. Alcuni economisti hanno notato che una spiegazione della complessità regolativa non ha bisogno di basarsi sugli interessi specifici dei regolatori, ma può fondarsi sugli interessi economici di parti terze, ovverosia degli specialisti nei vari aspetti regolativi, come avvocati, contabili, ingegneri o esperti di sicurezza. A differenza degli altri gruppi di interesse, tali esperti si preoccupano molto più del processo che del prodotto della regolazione, e traggono vantaggio dalla complessità regolativa, che accresce il valore della loro expertise. Così, gli intralci burocratici possono non essere semplicemente indice di inefficienza $o$ incapacità dell'amministrazione. Almeno in parte, sono il frutto di interessi privati che si giovano del fatto che un ambiente regolativo complesso permette agli specialisti di dominare i processi di definizione e di «intermediazione» delle norme (Kearl 1983; Quandt 1983).

Nel 1985 la Commissione ha introdotto un nuovo approccio alla regolazione tecnica con l'esplicito obbiettivo di ridurre la complessità regolativa (COM 1985/315, finale). In sostanza, il nuovo approccio propone una distinzione concettuale tra le materie dove l'armonizzazione delle normative nazionali è essenziale e quelle che possono essere lasciate nella sfera delle norme tecniche volontarie (dove vale il principio del «riferimento agli standard»), o dove è sufficiente un mutuo riconoscimento dei requisiti richiesti dalle leggi nazionali. In pratica il nuovo approccio rimpiazza una moltitudine di standard di specificazione (chiamati anche standard di processo o «ingegneristici») con pochi standard di performance che un prodotto deve soddisfare al fine di assicurare il diritto di libero movimento nel mercato unico europeo.

Le specificazioni tecniche formulate dagli organismi europei di standardizzazione (come il Comitato per la Standardizzazione Europea o il Comitato di standardizzazione dei prodotti elettrici) non sono obbligatorie e hanno sostanzialmente un carattere di standard volontari. Tuttavia i governi sono obbligati a presumere che i prodotti realizzati secondo gli standard europei 
(o, temporaneamente, secondo gli standard nazionali quando quelli europei non sono ancora disponibili) si conformino ai «requisiti fondamentali» ovvero agli standard di performance stipulati nella direttiva (Pelkmans 1987). Il sistema è completato dal mutuo riconoscimento delle procedure di collaudo e certificazione.

La nuova metodologia rivela un approccio altamente innovativo al problema generale della regolazione sovranazionale ed in particolare a quello della complessità regolativa, ma il suo successo dipende in maniera cruciale dal livello di reciproca fiducia tra gli stati membri. Senza di essa, infatti, i regolatori nazionali possono alterare il delicato equilibrio tra gli obbiettivi differenti e potenzialmente conflittuali (in particolare da un lato l'esigenza di assicurare il rispetto dei requisiti essenziali sanitari e di sicurezza e dall'altro quello di prevenire, attraverso la regolazione, la creazione di barriere interne al mercato) che sono gli elementi sui quali si basa il mutuo riconoscimento.

L'esperienza fatta con il mutuo riconoscimento dei criteri di ammissibilità di nuovi farmaci fornisce un buon esempio a questo proposito. Per oltre venti anni la Commissione ha tentato di armonizzare i regolamenti nazionali sui nuovi farmaci proponendo una serie di criteri per la verifica dei nuovi prodotti e per il mutuo riconoscimento dei test clinici e tossicologici condotti secondo le regole comunitarie. Secondo la «procedura sovranazionale per il riconoscimento dei farmaci» (MSAP) introdotta nel 1975, una impresa che ha ricevuto l'autorizzazione a commerciare da parte dell'agenzia regolativa di uno stato membro può chiedere il mutuo riconoscimento di tale autorizzazione almeno in altre cinque nazioni. Le agenzie delle nazioni chiamate in causa dall'impresa devono approvare o sollevare obbiezioni entro 120 giorni. In caso di obbiezioni, il Comitato sulla produzione di medicinali protetti da brevetto (CPMP), che include esperti nazionali e rappresentanti della Commissione, deve essere informato. Tale comitato deve poi esprimere la propria opinione entro 60 giorni, e nei successivi 30 giorni il suo giudizio può essere annullato dall'agenzia nazionale che aveva sollevato obbiezioni.

Purtroppo tale procedura non ha funzionato bene: i regolatori nazionali non si sentivano in alcun modo vincolati né dagli altri organi regolativi, né tantomeno dalle opinioni del CPMP. Anche una nuova procedura semplificata, introdotta nel 1983, non riuscì a rendere il processo più funzionale, poiché i regola- 
tori nazionali continuavano a sollevare sistematicamente obbiezioni reciproche (Kaufer 1990).

Queste difficoltà convinsero infine la Commissione a proporre la creazione di una agenzia europea per la valutazione dei prodotti medicinali e quindi una nuova procedura centralizzata a livello comunitario (obbligatoria per i prodotti bio-tecnologici e per alcuni tipi di farmaci di veterinaria, e opzionale per gli altri prodotti) che sanciscono una autorizzazione comunitaria (Commissione Europea 1990).

Come mostra questo esempio, un maggior livello di decentralizzazione sarebbe possibile qualora $\mathrm{i}$ regolatori nazionali si fidassero di più l'uno dell'altro. Spesso la sfiducia riflette una insufficiente conoscenza delle differenti filosofie regolative e degli stili nazionali di policy-making. Tuttavia in qualche caso $i$ regolatori nazionali hanno una bassa credibilità internazionale perché mancano, o quantomeno sono considerati carenti dagli interlocutori, dell'expertise tecnica e scientifica, delle risorse finanziarie e delle infrastrutture di policy necessarie per affrontare efficacemente problemi regolativi complessi. In tali casi, l'assistenza comunitaria può essere necessaria per assicurare che tutti i membri possano raggiungere un livello di competenza sufficiente per garantire la fiducia reciproca e rendere il mutuo riconoscimento possibile.

Il nocciolo della tesi qui esposta è in buona sostanza il fatto che i governi e le autorità regolative nazionali detengono una considerevole quota di responsabilità per il volume e la complessità della regolazione comunitaria. Perciò i rimedi dovrebbero essere cercati in primo luogo al livello degli stati membri, anche se un controllo centralizzato dei programmi regolativi all'interno della Commissione sarebbe naturalmente utile. L'imprenditorialità politica della Commissione diventa invece importante come fattore esplicativo per capire il progressivo «approfondimento della politica regolativa comunitaria».

$\mathrm{Nel}$ quarto e nel quinto paragrafo ho discusso in termini generali le condizioni che rendono possibile l'imprenditorialità nelle politiche pubbliche e le principali strategie seguite dagli «imprenditori» di successo. Ora vorrei considerare un aspetto particolare ma significativo del fenomeno di «approfondimento» della regolazione europea: il fatto che alcuni dei più evidenti esempi di innovazione nelle politiche comunitarie appartengono al settore della regolazione sociale (vedi secondo paragrafo). $\mathrm{Na}$ turalmente esiste una spiegazione semplice per questo fenome- 
no. Il livello di innovazione è infatti più limitato quando le politiche sono prescritte dai trattati - è il caso delle politiche pubbliche riguardanti la competizione, l'agricoltura o il commercio - o rappresentano una risposta obbligata ai bisogni funzionali di un mercato sempre più integrato - è il caso delle regole circa il libero movimento di beni, servizi, capitali e persone. Almeno fino all'Atto Unico Europeo la regolazione sociale non necessitava di essere giustificata in termini funzionali, e questo lasciava uno spazio maggiore all'imprenditorialità e all'innovazione rispetto alle tradizionali politiche pubbliche comunitarie.

Tuttavia, una spiegazione più interessante ci viene suggerita dalla ben nota tipologia delle politiche regolative proposta da James Q. Wilson, sulla base della distribuzione dei costi e dei benefici $(1980,366-372)$. In questa tipologia l'imprenditorialità politica corrisponde a quelle politiche pubbliche che conferiscono benefici diffusi (anche se piccoli) con costi a carico di un piccolo segmento della società.

Molte delle regolazioni sociali rientrano in questa categoria. Il costo di aria o di acqua più pulita, quello di prodotti più sicuri ed anche quello di migliori condizioni nell'ambiente di lavoro sono pagati, almeno inizialmente, da segmenti particolari dell'industria. Poiché l'incentivo ad organizzarsi nella difesa dei propri interessi è forte negli oppositori di tali politiche ma debole per i suoi beneficiari, simili misure regolative possono essere approvate soltanto se un imprenditore politico riesce a mobilitare l'opinione pubblica e ad associare la regolazione proposta con valori più ampi e condivisi, costringendo così gli oppositori sulla difensiva.

Secondo Wilson, l'imprenditore di policy è il rappresentante vicario di gruppi che non prendono parte direttamente al processo legislativo. Questa osservazione aiuta a capire la crescente importanza della regolazione sociale, e quella dell'imprenditorialità politica nella Comunità Europea. Storicamente, gli interessi diffusi sono stati scarsamente rappresentati in Europa poiché le forme tradizionali di intervento statale tendevano a favorire gli interessi dei produttori (capitalisti e lavoratori sindacalizzati) a discapito di quelli dei consumatori. Inoltre, i sistemi politici caratterizzati da un forte controllo partitico sia sull'esecutivo che sul legislativo e da una burocrazia pubblica fortemente centralizzata impedivano l'emergere di imprenditori politici indipendenti.

D'altra parte, l'isolamento della Commissione, un'istituzione non-maggioritaria, rispetto alla politica partitica, l'attivismo del- 
la Corte Europea di Giustizia e gli sforzi del Parlamento Europeo per individuare un proprio ruolo specifico sono tutti fattori che spiegano perché gli interessi diffusi siano spesso protetti in modo più efficace al livello europeo che non a quello nazionale. I critici della crescita regolativa nella Comunità Europea non devono dimenticare che in molti stati membri la legislazione di protezione del consumatore ed anche le politiche ambientali erano scarsamente sviluppate, se non completamente assenti, prima che i governi nazionali venissero obbligati ad applicare le direttive europee relative a questi settori.

\section{Verso le riforme istituzionali}

Gli osservatori sono sostanzialmente concordi nell'affermare l'impossibilità di applicare le attuali regole di funzionamento ad una Comunità con 16 membri o ancora di più: la necessità di riforme istituzionali è dunque urgente e spesso evocata. Sebbene il presente saggio non si occupi specificamente di questo tema alcune delle conclusioni raggiunte possono essere rilevanti in questa prospettiva.

Un primo punto da rilevare è di natura metodologica: alla luce di quanto detto non si dovrebbe enfatizzare la natura sui generis della Comunità ma piuttosto tentare di distinguere tra $\mathrm{i}$ problemi idiosincratici e quelli che possono essere ricondotti in via generale ad uno stile specifico di policy-making o ad un metodo di governo. In questa ottica si può osservare che la sovraregolazione è un problema generale, sebbene essa possa essere aggravata da particolari meccanismi istituzionali e politici propri della Comunità. Da ciò si evince che le proposte di riforma non dovrebbero essere concepite ad boc, ma sulla base di principi ispiratori generali.

Questo si applica anche ad un problema politico fondamentale, che è quello del «deficit democratico» delle istituzioni europee ed in particolare della Commissione. Come ho sottolineato altrove, un problema di responsabilità democratica sopravviene quando importanti poteri di policy-making sono delegati a istituzioni non rappresentative come le banche centrali politicamente indipendenti e le commissioni regolative. Discutendo il problema esclusivamente nel contesto della Comunità Europea si corre il rischio di trascurare esperienze nazionali rilevanti e di adottare soluzioni ad boc potenzialmente inefficaci. 
In secondo luogo, la discussione precedente sulla sovra-regolazione e sulla complessità regolativa ci suggerisce che è inutile, ed anche non corretto, rimproverare alla Commissione tutte le disfunzioni del policy-making a livello europeo. Se infatti è vero che gli stati membri hanno la loro quota di responsabilità, allora le riforme istituzionali devono cominciare proprio da loro. Uno dei temi centrali di questo saggio è l'importanza determinante della fiducia reciproca tra gli stati membri. Abbiamo visto che il mutuo riconoscimento non può avere successo quando i regolatori nazionali mostrano reciproca sfiducia. Ma problemi non dissimili emergono anche nelle applicazioni pratiche del principio di sussidiarietà. Questo perché i governi nazionali e sub-nazionali, che sono certamente più in sintonia con le preferenze individuali dei cittadini, sono tuttavia poco portati a distinguere con precisione tra una mera attività di produzione di beni pubblici e l'impegnarsi in politiche pubbliche disegnate per avvantaggiare il proprio paese o la propria regione rispetto ai vicini.

Per esempio, le autorità locali hanno a volte tentato di controllare l'inquinamento atmosferico imponendo alle industrie ciminiere altissime. In tal modo, le emissioni discendono al suolo ad una certa distanza, generalmente in una città, una regione o nel paese vicino, cioè fuori della giurisdizione dell'autorità che ha imposto quella norma.

Finché i regolatori non sviluppano quella fiducia reciproca che consenta loro di evitare strategie egoistiche di questo tipo, la centralizzazione dell'autorità regolativa è l'unica soluzione pratica per correggere la tendenza a spostare le esternalità oltre i confini o per impedire che l'attività locale regolativa di un fallimento del mercato si trasformi in una barriera per il libero scambio.

Un ultimo punto di riflessione riguarda le procedure di policy-making. Come si è già detto in precedenza, le attività regolative della Commissione sono sostenute da un ampio network di comitati consultivi, regolativi e di gestione. Inoltre, a fine ottobre 1993, per decisione degli stati membri, sono stati creati e localizzati dieci nuovi organismi amministrativi. Tra questi, oltre all'Istituto Monetario Europeo di Francoforte, che è il precursore della Banca Centrale Europea, ci sono l'Agenzia Europea per l'Ambiente, l'Ufficio per l'Ispezione ed il Controllo Veterinario e Fitosanitario, il Centro Europeo per il controllo della Droga e della Tossicodipendenza, l'Agenzia Europea per la 
valutazione dei Prodotti Farmaceutici e l'Agenzia per l'Igiene e la Sicurezza nei posti di lavoro.

Questa proliferazione di comitati tecnici e di agenzie specializzate aggrava uno dei più preoccupanti difetti della struttura istituzionale comunitaria: la mancanza di trasparenza nei processi decisionali. A causa dell'opacità delle procedure, è difficile per i cittadini dell'Unione identificare l'organo responsabile delle decisioni che li riguardano ed i rimedi legali disponibili.

Una situazione simile accadde negli Stati Uniti al tempo del New Deal, quando si verificò una drammatica crescita nell'intervento statale. La nascita di nuove agenzie specializzate, le cui funzioni erano estremamente complesse e diverse, creò il bisogno di nuove norme per assicurare che esse non agissero arbitrariamente od in modo illegale. Nell'assenza di una vera e propria tradizione di diritto amministrativo, la normativa sulla amministrazione federale era cresciuta in modo frammentario e non coordinato, in risposta di volta in volta ad esigenze specifiche. Tuttavia, questo approccio fu giudicato insufficiente per fronteggiare $\mathrm{i}$ cambiamenti che stavano realizzandosi in quel periodo. L'Administrative Procedures Act (APA), approvato dal Congresso nel 1946, mirò a legittimare la crescita della burocrazia federale, stabilendo un insieme unitario di regole per definire le procedure che le agenzie federali avrebbero dovuto seguire e prevedendo il controllo giurisdizionale su molte delle decisioni di queste.

Mi pare che la Comunità Europea potrebbe trarre utilmente insegnamento da questo precedente. L'emanazione di una legge sulle procedure amministrative a livello europeo fornirebbe alla Comunità una occasione unica per decidere che tipo di regole sono preferibili per la razionalizzazione del processo decisionale, quale accesso debbano avere i gruppi di interesse nei processi regolativi ed anche per stabilire quando è necessario un controllo giurisdizionale. Anche se si trattasse soltanto di codificare in forma legislativa le prassi esistenti, come fece in larga misura l'APA americano, l'adozione di un unico corpo di regole amministrative potrebbe perlomeno fornire un nucleo centrale di strumenti applicabili alle sempre più estese attività regolative. Un passo di questo genere testimonierebbe, inoltre, la volontà della Comunità e degli stessi stati membri di non accettare la crescita incontrollata delle funzioni amministrative della Comunità. 


\section{Riferimenti bibliografici}

Alter, K.J. e S.M. Meunier-Aitsahalia (1993), Judicial Politics in the European Community: European Integration and the Pathbreaking Cassis de Dijon Decision, paper presentato alla Conferenza dell'Associazione Studi sulla Comunità Europea, Washington D.C., 27-29 maggio 1993.

Coase, R. (1960), The Problem of Social Cost, in «The Journal of Law and Economics», 3, pp. 1-44.

Commissione Europea (1985), Completing the Internal Market, COM (85) 310, final, Luxembourg, Office for Official Pubblications of the European Communities.

Commissione Europea (1990), Future System for the Free Movement of Medicinal Drugs in the European Community, COM (90) 283 final, Luxembourg, Office for Official Publications of the European Communities.

Conseil d'Etat (1992), Rapport public 1992, Paris, La documentation Française, Etudes et Documents.

Dehousse, R. (1994), La Cour de Justice des Communautés Européennes, Paris, Montchretien.

De Muth, C. (1984), A Strategy for Regulatory Reform, in «Regulation», 4, pp. 25-30.

Eichener, V. (1992), Social Dumping or Innovative Regulation?, Florence, European University Institute, Working Paper SPS n. 92/ 28.

Feldhoff, K. (1992), Grundzüdes Europäischen Arbeitsum-weltrechts, mimeo, Bochum, Rurhr Universität.

Garret, G. (1992), International Cooperation and Institutional Choiche: The European Community's Internal Market, in «International Organization», 46, pp. 533-560.

Garret, G. e B. Weingast (1993), Ideas, Interests and Institutions: Constructing the European Community Internal Market, in J. Goldestein e R. Kehoane (a cura di), Ideas and Foreign Policy, Ithaca, N.Y., Cornell University Press.

Gatsios, K. e P. Seabright (1989), Regulation in the European Community, in «Oxford Review of Economic Policy», 5, no. 2, pp. 37-60.

George, S. (1993), Supranational Actors and Domestic Politics: Integration Theory Reconsidered in the Light of the Single European Act and Maastricht, Sheffield, mimeo.

Haas, E. (1958), The Uniting of Europe: Political, Social and Economic Forces, 1950-1957, Stanford, Stanford University Press.

Institut fuer Europaeische Politik (1989), «Comitology»: Charactheristics, Performance and Options, Bonn Preliminary Final Report.

Kaufer, E. (1990), The Regulation on New Product Development in the Drug Industry, in G. Majone (a cura di), Deregulation or Re-regulation?, London, Pinter Publishers, pp. 223-251. 
Kearl, J. (1983), Rules, Rule Intermediaries and the Complexity and Stability of Regulation, in «Journal of Public Economics», 22, pp. 215-226.

Keohane, R. e S. Hoffman (1990), Community Policy and Institutional Change, in W. Wallace (a cura di), The Dynamics of European Integration, London, Pinter Publishers.

Kingdom, J.W. (1984), Agendas, Alternatives and Public Policy, Boston, Little Brown.

Ludlow, P. (1991), The European Commission, in R. Keohane e S. Hoffman (a cura di), The New European Community: Decision Making and Institutional Change, Boulder, CO, Westview Press, pp. 85-132.

Majone, G. (1992), Market Integration and Regulation: Europe after 1992, in «Metroeconomica», 43, 1-2, pp. 131-156.

- (1994a) The Rise of the Regulatory State in Europe, in «West European Politics», 17, 3, pp. 78-102.

- (1994b) Independence vs. Accountability? Non-majoritarian Institutions and Democratic Government in Europe, in J.J. Hesse (a cura di), The European Yearbook on Comparative Government and Public Administration, Oxford, Oxford University Press.

- (1994c) Controlling Regulatory Bureaucracies: Lessons from the American Experience, in H.U. Derlien, U. Gerhardt, F.W. Scharpf (a cura di), Systemrationalitaet und Partialinteresse, Baden-Baden, Nomos, pp. 291-314.

Mayhew, D.R. (1974), Congress: the Electoral Connection, New Haven, Yale University Press.

Moe, T.M. (1987), The Politics of Structural Choice: toward a Theory of Public Bureaucracy, in O.E. Williamson (a cura di), Organization Theory from Chester Barnard to the Present, Oxford, Oxford University Press, pp. 116-153.

Moravcsik, A. (1993), Preferences and Power in European Community: a Liberal Intergovernalistic Approach, in «Journal of Common Market Studies», 31, 4, pp. 473-524.

Peacock, A. (1984), The Regulation Game, Oxford, Basil Blackwell.

Pelkmans, J. (1987), The New Approach to Technical Harmonization and Standardization, in «Journal of Common Market Studies», 25, 3 pp. 249-269.

Peters, G.B. (1992), Bureaucratic Politics and the Institutions of the European Community, in A.M. Sbragia (a cura di), Europolitics, Washington, The Brooking Institution.

Quandt, R.E. (1983), Complexity in Regulation, in «Journal of Public Economics», 22, pp. 199-214.

Rehbinder, E. e R. Stewart (1985), Environmental Protection Policy, Berlin, De Gruyter.

Riker, W. (1986), The Art of Political Manipulation, New Haven, Yale University Press. 
Wildavsky, A. (1964), The Budgetary Process, Boston, Little Brown. Williamson, O.E. (1985), The Economic Institutions of Capitalism, New York, The Free Press.

Wilson, J.Q. (1980), The Politics of Regulation, New York, Basic Books.

Wood, D.B. e R.W. Waterman (1991), The Dynamics of Political Control in the Bureaucracy, in «American Political Science Review», 85 , pp. 801-828. 